\title{
Quantum Anomalous Vortex and Majorana Zero Mode in Iron-Based Superconductor Fe(Te,Se)
}

\author{
Kun Jiang, ${ }^{1,2}$ Xi Dai, ${ }^{3}$ and Ziqiang Wang ${ }^{1}$ \\ ${ }^{1}$ Department of Physics, Boston College, Chestnut Hill, Massachusetts 02467, USA \\ ${ }^{2}$ Beijing National Laboratory for Condensed Matter Physics and Institute of Physics, \\ Chinese Academy of Sciences, Beijing 100190, China \\ ${ }^{3}$ Department of Physics, Hong Kong University of Science and Technology, Kowloon, Hong Kong
}

(Received 22 October 2018; revised manuscript received 28 December 2018; published 19 February 2019)

\begin{abstract}
In topological insulators doped with magnetic ions, spin-orbit coupling and ferromagnetism give rise to the quantum anomalous Hall effect. Here, we show that in $s$-wave superconductors with strong spin-orbit coupling, magnetic impurity ions can generate topological vortices in the absence of external magnetic fields. Such vortices, dubbed quantum anomalous vortices, support robust Majorana zero-energy modes when superconductivity is induced in the topological surface states. We demonstrate that the zero-energy bound states observed in $\mathrm{Fe}(\mathrm{Te}, \mathrm{Se})$ superconductors are possible realizations of the Majorana zero modes in quantum anomalous vortices produced by the interstitial magnetic Fe. The quantum anomalous vortex matter not only advances fundamental understandings of topological defect excitations of Cooper pairing but also provides new and advantageous platforms for manipulating Majorana zero modes in quantum computing.
\end{abstract}

DOI: 10.1103/PhysRevX.9.011033

\section{INTRODUCTION}

Harvesting localized Majorana fermion excitations has thrived in condensed matter and materials physics for both its fundamental value and its potential for faulttolerant non-Abelian quantum computing [1-11]. An important and promising path discovered thus far is to combine spin-orbit coupling (SOC) and the Berry phase of the electrons with superconductivity. Localized Majorana zero-energy modes (MZM) have been proposed to arise in the vortex core when the Dirac fermion surface states of a topological insulator are proximity coupled to an $s$-wave superconductor [5], or when superconductivity is induced in a semiconductor with strong Rashba SOC and a time-reversal symmetry-breaking Zeeman field [6]. Experimental realizations of these proposals are under active current investigations [12-15]. However, there exists fundamental challenges that come with using external magnetic-field-induced vortices. The existence and the stability of the MZM in real materials are not guaranteed due to the low-energy vortex core states $[5,16,17]$ as well as disorder and vortex creep. It is

Published by the American Physical Society under the terms of the Creative Commons Attribution 4.0 International license. Further distribution of this work must maintain attribution to the author(s) and the published article's title, journal citation, and DOI.
Subject Areas: Condensed Matter Physics,

Quantum Physics, Superconductivity difficult, if not impossible, to move the field-induced vortex lines individually on the Abrikosov lattice, which greatly reduces the ability to manipulate the MZM for operations such as braiding. Moreover, the requirement of the external field is difficult to integrate into quantum computation devices and limits their applications.

We propose here a new form of vortex matterthe quantum anomalous vortex matter that can support robust MZM without applying an external magnetic field. In conventional spin-singlet $s$-wave superconductors, a time-reversal symmetry-breaking magnetic impurity is known to create a vortex-free defect hosting the YuShiba-Rusinov (YSR) bound states [18-20] inside the superconducting (SC) gap. We find that this folklore changes in a fundamental way in $s$-wave superconductors with strong SOC. In this case, topological defect excitations can be generated by a quantized phase winding of the SC order parameter around the magnetic impurity, all without applying an external magnetic field. The role of the magnetic field is played by the combination of the exchange field and SOC as in the anomalous Hall effect. The emergence of such vortices is thus remarkably analogous to the quantum anomalous Hall effect in topological insulator thin films doped with magnetic ions [21,22] hence the term quantum anomalous vortex (QAV). We demonstrate with theoretical calculations that (i) the QAV nucleates around the magnetic ion by the exchange coupling between the local moment and spin-angularmomentum-locked SC quasiparticles that lowers its energy 


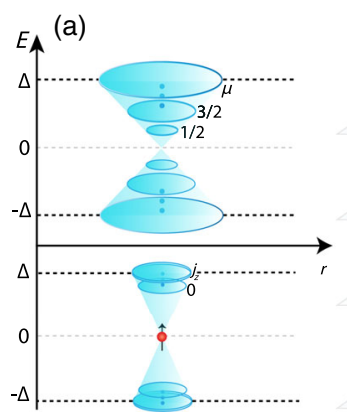

(b)

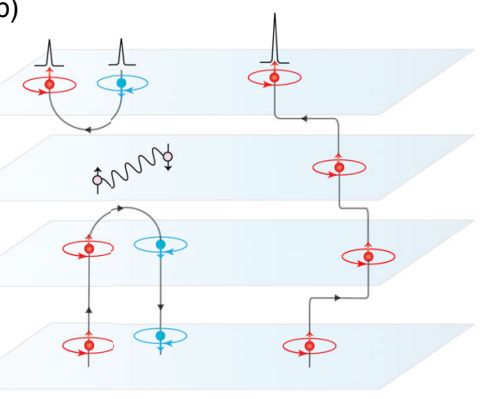

FIG. 1. Schematic rendering of (a) a conventional magneticfield-induced vortex (top panel) and the QAV nucleated at a magnetic ion (bottom panel), showing energy levels of the in-gap CdGM states localized inside the vortex core and labeled by the quantum numbers $\mu$ and $j_{z}$, respectively. Negative energy states are occupied. (b) Schematic rendering of the quantum anomalous vortex matter in the layered superconductor. Red and blue dots or arrows indicate opposite $c$-axis moment directions of the magnetic ions, while arrowed circles represent the vorticities of the QAV. Black lines are the continuous flux lines piercing SC layers through the magnetic ions. Zero-bias peaks indicate the localized MZM from the TSS in the QAV cores where the flux lines enter and leave the sample surface.

compared to the vortex-free YSR state; (ii) when superconductivity is induced in the topological surface states, MZM emerge inside the QAV core, again without applying an external magnetic field; (iii) a remarkable property of the QAV is that the Caroli-de Gennes-Matricon (CdGM) vortex core states $[23,24]$ with nonzero effective angular momenta are expelled into the continuum above the SC gap. A comparison of the vortex profile and core states between the QAV and the field-induced vortex is shown in Fig. 1(a). The "gapping" of the core states critically enhances the stability and robustness of the MZM by preventing the mixing with the CdGM states at nonzero energy $[5,16,17]$. At low densities of magnetic ions, a new electronic matter, the QAV matter with surface MZM as depicted in Fig. 1(b), would arise in such layered superconductors and provide an unprecedented platform of robust and manipulatable MZM for non-Abelian quantum computing.

We find that the QAV matter is pertinent to the Fe-based superconductor $\mathrm{Fe}(\mathrm{Te}, \mathrm{Se})$, exhibiting spectroscopic properties remarkably consistent with the surprising discovery of robust zero-energy bound states (ZBS) near the excess Fe by STM in the absence of external magnetic fields [25]. Topological surface states (TSS) in $\mathrm{Fe}(\mathrm{Te}, \mathrm{Se})[26,27]$ have been observed by ARPES recently and acquire a SC gap below $T_{c}$ by the natural coupling to bulk superconductivity in the same crystal [28]. The condition for the applied magnetic-field-induced vortices to host ZBS is still unclear, with one group reporting its absence and CdGM vortex core states at nonzero energies [29] and another finding the ZBS in about $20 \%$ of the vortices [30]. However, the observation of ZBS at all excess Fe sites in zero field is ubiquitous with measured properties fully consistent with

MZM [25]. The excess $\mathrm{Fe}$ in as-grown $\mathrm{Fe}(\mathrm{Te}, \mathrm{Se})$ are native magnetic impurities sitting at the $C_{4}$ symmetric interstitial site surrounded by the Te atoms responsible for the strong SOC. Neutron scattering finds that each excess Fe carries a $c$-axis local magnetic moment $\left(\sim 2.5 \mu_{B}\right)$ and induces a ferromagnetic cluster in the neighboring Fe sites [31]. We show by explicit calculations that the ZBS are consistent with MZM localized in the QAV induced by the interstitial Fe with the crucial caricature of the expulsion of nonzero energy CdGM states observed in the STM data [25].

\section{THEORETICAL MODEL}

The bulk and surface electronic structures of $\mathrm{Fe}(\mathrm{Te}, \mathrm{Se})$ have been studied in recent theoretical works $[16,26,27]$. In a nutshell, with increasing Te concentration, the band derived from the $\mathrm{Te} / \mathrm{Se} p_{z}$ orbital is pushed down in energy and hybridizes strongly with the Fe $d$ orbitals. The intrinsic SOC enhanced by Te then opens up a gap and induces a $p-d$ band inversion near the $Z$ point in the Brillouin zone, giving rise to the TSS upon projection onto the (001) surface. The situation is analogous to a 3D topological insulator, with the exception that the normal state of $\mathrm{Fe}(\mathrm{Te}, \mathrm{Se})$ is a metal and the TSS is electron doped. The bulk and the surface electronic structures have recently been qualitatively confirmed by the high-resolution, spin-polarized ARPES experiments [28,32].

Here, we construct an effective low-energy theory where the bands of the bulk and surface states are treated separately in an $s$-wave superconductor with intrinsic SOC. An isolated magnetic ion, such as the interstitial $\mathrm{Fe}$ impurity in $\mathrm{Fe}(\mathrm{Te}, \mathrm{Se})$, is introduced at $\mathbf{r}=0$. Our strategy is to first study the bulk SC state and show that a topological defect excitation, where the phase of the pairing order parameter winds by $2 \pi$ as in a vortex, nucleates spontaneously at the magnetic ion. This part is independent of the existence of TSS. Then, we couple such a novel QAV to the helical Dirac fermion TSS and study the emergence of the robust MZM. Using $\mathrm{Fe}(\mathrm{Te}, \mathrm{Se})$ as a reference material, we consider a holelike bulk band around the $\Gamma$ point as shown in Fig. 2(a), with the effective mass $m^{*} \simeq 4.08 m_{e}$ and Fermi energy $\varepsilon_{f} \simeq-4.52 \mathrm{meV}$ extracted from the ARPES experiments [28]. The role of the electronlike band in $\mathrm{Fe}(\mathrm{Te}, \mathrm{Se})$ will be discussed later. In the spinor basis $\psi(\mathbf{r})=\left[\psi_{\uparrow}(\mathbf{r}), \psi_{\downarrow}(\mathbf{r})\right]^{T}$, the normal state Hamiltonian reads, in the continuum limit,

$$
H=-\frac{p^{2}}{2 m^{*}}-\varepsilon_{f}+H_{\mathrm{soc}}+H_{\mathrm{ex}} .
$$

Here, the SOC term is described by

$$
H_{\mathrm{soc}}=-\lambda_{\mathrm{so}}(r) \mathbf{L} \cdot \boldsymbol{\sigma},
$$

where $\mathbf{L}=\mathbf{r} \times \mathbf{p}$ is the angular-momentum operator and $\boldsymbol{\sigma}$ the vector spin Pauli matrix. Note that, in the presence of the intrinsic SOC $[26,27]$, the spin rotation symmetry is broken and the band electrons carry the pseudospin quantum number. 

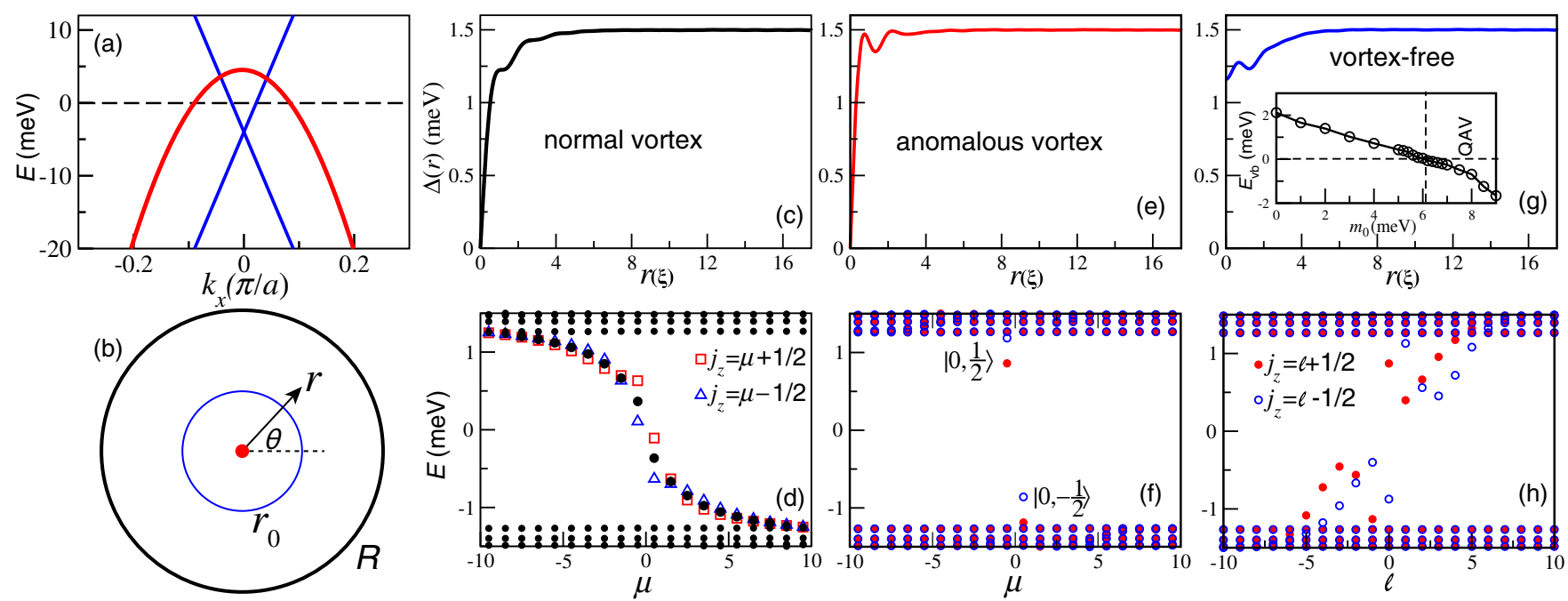

FIG. 2. (a) The effective holelike band for bulk states (red line) and the TSS band (blue line) observed in Ref. [28] near the $\Gamma$ point. (b) The disc geometry with radius $R$. Short-ranged couplings have an exponential decay length $r_{0}$ from the centered magnetic ion. (c)-(h) The self-consistent pairing profile $\Delta(r)$, where $r$ is measured in units of the coherence length $\xi$ and the in-gap bound-states spectrum: (c,d) The normal magnetic-field-induced vortex. The SOC associated with the magnetic ion with $\lambda_{0}=6.6$ meV splits the degenerate CdGM states $|\mu, \sigma\rangle$ (black circles) into two sets of spin-orbit-coupled bound states $\left|j_{z}, \sigma\right\rangle$ with $j_{z}=\mu \pm \frac{1}{2}$ (open squares and triangles). (e,f) The QAV induced by the magnetic ion for $m_{0}=\lambda_{0}=6.6 \mathrm{meV}$. All CdGM states $\left|j_{z}, \sigma\right\rangle$ except $|0, \sigma\rangle$ are pushed to higher binding energies above the SC gap. $(\mathrm{g}, \mathrm{h})$ The vortex-free state near the magnetic ion. The SOC splits the in-gap YSR states into two sets. Inset in $(\mathrm{g})$ : the QAV binding energy as a function of the exchange field $m_{0}$. The QAV emerges beyond $m_{0}^{c} \simeq 6.1 \mathrm{meV}$.

Since it will not affect our results, we use the term spin instead of pseudospin for simplicity. The $\lambda_{\text {so }}(r)$ in Eq. (2) comes from the Elliot-Yafet SOC induced by the impurity [33,34] embedded in the strongly spin-orbit-coupled environment. The spin and angular momentum of the partial waves are thus locked by the impurity whose magnetic moment $\mathbf{I}_{\text {imp }}$ involves both spin and orbital contributions of the magnetic ion. The exchange interaction in Eq. (1) thus contains both spin and orbital exchange processes [35-39] and can be written in the basis of the total angular momentum $\mathbf{J}=\mathbf{L}+\frac{1}{2} \boldsymbol{\sigma}$ as

$$
H_{\mathrm{ex}}=-\mathcal{J}_{\mathrm{ex}}(r) \mathbf{I}_{\mathrm{imp}} \cdot \mathbf{J} .
$$

For simplicity, the short-ranged exchange coupling $\mathcal{J}_{\text {ex }}(r)$ is assumed to be isotropic to keep the parameters of the theory at a minimum. Equation (3) is similar to the exchange interaction in dilute magnetic semiconductors [40].

To study the SC state with a complex inhomogeneous Cooper-pairing order parameter, it is convenient to perform the Bogoliubov transformation

$$
\psi_{\sigma}^{\dagger}(\mathbf{r})=\sum_{n}\left[u_{n \sigma}^{*}(\mathbf{r}) \gamma_{n}^{\dagger}+v_{n \sigma}(\mathbf{r}) \gamma_{n}\right]
$$

where $\gamma_{n}^{\dagger}$ creates a Bogoliubov quasiparticle. The resulting Bogoliubov-de Gennes (BdG) equation is given by

$$
\left[\begin{array}{cc}
H & \boldsymbol{\Delta}(\mathbf{r}) \\
\boldsymbol{\Delta}^{*}(\mathbf{r}) & -\sigma_{y} H^{*} \sigma_{y}
\end{array}\right] \Phi_{n}(\mathbf{r})=E_{n} \Phi_{n}(\mathbf{r}),
$$

where $\boldsymbol{\Delta}(\mathbf{r})=g\left\langle\psi_{\downarrow}(\mathbf{r}) \psi_{\uparrow}(\mathbf{r})\right\rangle$ is the self-consistent pairing potential for an attraction $g$ [41]. We choose $g=11 \mathrm{meV}$ such that the calculated $|\Delta(\mathbf{r})|=\Delta=1.5 \mathrm{meV}$ matches the bulk SC gap determined experimentally for this band $[25,28]$. The BCS coherence length is therefore $\xi=$ $\hbar v_{f} / \pi \Delta \simeq 2.76 \mathrm{~nm}$, which is not far from the measured value of about $2 \mathrm{~nm}$ in $\mathrm{Fe}(\mathrm{Te}, \mathrm{Se})$ [42]. Diagonalizing the $\mathrm{BdG}$ equation yields the energy spectrum $E_{n}$ and Nambu wave functions $\Phi_{n}(\mathbf{r})=\left[u_{n \uparrow}(\mathbf{r}), u_{n \downarrow}(\mathbf{r}), v_{n \downarrow}(\mathbf{r}),-v_{n \uparrow}(\mathbf{r})\right]^{T}$ for both the vortex-free and vortex solutions with $\Delta(\mathbf{r})=\Delta(r) e^{i \nu \theta}$, where the integer $\nu$ is the vorticity.

We have obtained the solutions in the disc geometry for a SC layer with the isolated magnetic ion at its center [Fig. 2(b)] in polar coordinates $\mathbf{r}=(r, \theta)$. The SOC in Eq. (2) reduces to $-\lambda_{\mathrm{so}}(r) L_{z} \sigma_{z}$, with $L_{z}=-i \hbar \partial_{\theta}$. The wave function is factorizable according to

$$
\begin{array}{r}
\Phi_{n \mu}(r, \theta)=e^{i \mu \theta}\left[u_{n \mu+\frac{\nu}{2} \uparrow}(r) e^{i \frac{\nu}{2} \theta}, u_{n \mu+\frac{\nu}{2} \downarrow}(r) e^{i \frac{\nu}{2} \theta},\right. \\
\left.v_{n \mu-\frac{\nu}{2} \downarrow}(r) e^{-i \frac{i}{2} \theta},-v_{n \mu-\frac{\nu}{2} \uparrow}(r) e^{-i \frac{i}{2} \theta}\right]^{T},
\end{array}
$$

where the principal quantum number $n$ is determined by solving the radial $(u, v)$ in the basis of Bessel functions and the angular quantum number $\mu=\ell-(\nu / 2)$, with $\ell$ an integer $[23,24,41,43]$. The details of the calculation are given in the Supplemental Material [41]. The calculations are performed on discs of radius $R=87.5 \xi$. The SOC and exchange coupling due to the magnetic ion are assumed to decay exponentially, $\lambda_{\text {so }}(r), \mathcal{J}_{\text {ex }}(r) \propto e^{-r / r_{0}}$, with a common 
decay length $r_{0}=0.7 \xi$ [Fig. 2(b)] for simplicity and easy comparison.

\section{NORMAL VORTEX STATE}

In the absence of the magnetic ion, the self-consistent pairing profile $\Delta(r)$ for the field-induced vortex solution with $\nu=-1$ is shown in Fig. 2(c). It vanishes at the vortex center, exhibits the characteristic Friedel-like oscillations $[43,44]$, and approaches the bulk value for $r>6 \xi$. The eigenstate energies are plotted in Fig. 2(d), zoomed in to the range $(-\Delta, \Delta)$ to display the in-gap CdGM vortex core states. These bound states are doubly degenerate, carry the half-integer quantum number $\mu$, and grow with $\mu$ initially as $E_{\mu} \propto \mu\left(\Delta^{2} / \varepsilon_{f}\right), \quad \mu= \pm \frac{1}{2}, \pm \frac{3}{2}, \ldots \quad[23,24]$. Owing to the small $\left|\varepsilon_{f}\right|$ and the large $\Delta$ in $\mathrm{Fe}(\mathrm{Te}, \mathrm{Se})$, the CdGM states are in the quantum limit $\left(\xi k_{f} \sim 2\right)$ with the onset at $E_{-\frac{1}{2}} \simeq 0.38 \mathrm{meV}$ [Fig. 2(d)]. The vortex binding energy is defined as

$$
E_{\mathrm{vb}}=E_{\mathrm{vortex}}-E_{\mathrm{vortex}-\text { free }},
$$

where $E_{\text {vortex }}$ and $E_{\text {vortex-free }}$ are the energy of the vortex and the energy of the vortex-free state, respectively. There is an energy cost $\left(E_{\mathrm{vb}}>0\right)$ for creating the normal vortex since the supercurrent-carrying midgap CdGM states with $E_{\mu}<0$ are occupied. An external magnetic field must therefore be applied to break the time-reversal symmetry and provide the energy cost for creating the vortex cores and the magnetic flux lines in order to support such topological defect excitations in usual superconductors.

Note that there exists a sense of "chirality" for the vortex core states shown in Fig. 2(d), i.e., $\mu E_{\mu}<0$, which is determined by the particle-hole vorticity $\operatorname{sgn}\left(\varepsilon_{f}\right) \nu>0$. It can be flipped by either changing the sign of $\nu$ (controlled by the direction of the external magnetic field for normal vortices) or by changing to an electron band with $\varepsilon_{f}>0$. As we show below, for the QAV nucleated at the magnetic ion of a given moment polarization direction, this chirality is determined by the condition to lower the vortex binding energy with a spontaneously determined sign of the vorticity $\nu$.

\section{QUANTUM ANOMALOUS VORTEX STATE}

Let us switch on the SOC due to the magnetic ion, $H_{\mathrm{soc}}=-\lambda_{\mathrm{so}}(r) L_{z} \sigma_{z}$, with $\lambda_{\mathrm{so}}(r)=\lambda_{0} e^{-r / r_{0}}$ in Eq. (2). It splits off in energy the nonzero angular-momentum partial waves with different spin- $\sigma_{z}$ projections [41]. While $\lambda_{0}$ is not known directly from current experiments, the SOCinduced band splittings in bulk $\mathrm{FeSe}$ and $\mathrm{Fe}(\mathrm{Te}, \mathrm{Se})$ have been measured by ARPES to be in the range of $20-40 \mathrm{meV}$ $[45,46]$. We expect $\lambda_{0}$ to be in a similar range. Considering the effective nature of the theory, we use a smaller value $\lambda_{0}=6.6 \mathrm{meV}$ of no particular significance for the electrons in the holelike band in the rest of the paper, unless otherwise noted. As shown in Fig. 2(d) by the colored symbols, each doubly degenerate CdGM state splits into $\left|j_{z}, \pm \frac{1}{2}\right\rangle$ by an amount controlled by $\lambda_{0}$. Because $\lambda_{\text {so }}(r)$ decreases exponentially, the higher orbital angular-momentum $\ell$ states with wave functions concentrated further away from the magnetic ion experience a smaller $\lambda_{\mathrm{so}}(r)$. As a result, the SOC effect on the vortex core states is most pronounced for the small $\mu$ CdGM states. Moreover, the nonmonotonic SOC energy of the $\ell$ states leads to a sign change in the splitting of the $\left|j_{z}, \pm \frac{1}{2}\right\rangle$ states at larger $\mu$, as can be seen in Fig. 2(d). Since all the negative-energy CdGM states are occupied, SOC alone does not significantly lower the vortex core energy.

The binding energy of the QAV comes from the exchange interaction in Eq. (3) under SOC, which is qualitatively different from the proposal of a spontaneous vortex lattice in ferromagnetic superconductors [47]. Since magnetic transition metal ions usually have a large moment, such as the excess $\mathrm{Fe}$ moment in $\mathrm{Fe}(\mathrm{Te}, \mathrm{Se})$ [31] pointing along the $c$ axis due to the magnetic anisotropy, the impurity moment can be treated classically, i.e., $\mathbf{I}_{\text {imp }}=M \hat{z}$. The exchange interaction becomes $H_{\mathrm{ex}}=-m(r) J_{z}$, with $m(r)=\mathcal{J}_{\mathrm{ex}}(r)$ $M \equiv m_{0} e^{-r / r_{0}}$. Consequently, for $m_{0}>0$, the energy of the CdGM states shown in Fig. 2(d) is lowered (raised) by the spin-orbit exchange field $-m(r) j_{z}$ for all positive (negative) $j_{z}$. This expulsion of the in-gap CdGM vortex core states toward the continuum above the SC gap is the crucial mechanism for lowering the core energy of the QAV by the exchange field. For a local moment polarized in the opposite direction, the exchange field changes a sign, i.e., $m_{0}<0$; the vortex core energy can be lowered in the same manner if the QAV nucleates with an opposite particlehole vorticity $\operatorname{sgn}\left(\varepsilon_{f}\right) \nu<0$ and a flipped chirality of the vortex core states $\mu E_{\mu}>0$.

Figure 2(e) shows the self-consistent vortex profile calculated using the full Hamiltonian $H$ with a $m_{0}=6.6 \mathrm{meV}$. The vortex core sharpens considerably, and the Friedel oscillations become more prominent compared to the normal field-induced vortex in Fig. 2(c). The eigenstate energies are plotted in Fig. 2(f). All but two of the CdGM states $\left|j_{z}, \sigma_{z}\right\rangle$ are expelled out of the gap center into the continuum. The remaining two states sitting just below the gap edges have $j_{z}=0$ and are thus unaffected by the exchange field. They could have been pushed further toward the continuum by a larger $\lambda_{0}$. The enhanced binding energy of the occupied CdGM states significantly lowers the energy of the anomalous vortex state. In contrast, the vortex-free $(\nu=0)$ state obtained self-consistently using the same parameters shows a much broader deformation in $\Delta(r)$ near the magnetic ion [Fig. 2(g)] and fosters two sets of spin-orbit-coupled midgap YSR states [Fig. 2(h)] that must be occupied at an energy cost. Thus, the energy of the quantum anomalous vortex state nucleated at the magnetic ion can be lower than that of the vortex-free state, provided that the exchange field $m_{0}$ is sufficient to drive $E_{\mathrm{vb}}<0$. 
We next calculate the vortex binding energy as a function of $m_{0}$, which is shown in the inset of Fig. 2(g). Note that $E_{\mathrm{vb}}$ decreases approximately linearly with increasing $m_{0}$, and a transition from the vortex-free YSR state to the QAV state occurs at a critical $m_{0}^{c} \simeq 6.1 \mathrm{meV}$. For $m_{0}>m_{0}^{c}$, $E_{\mathrm{vb}}<0$, and it becomes more energetically favorable for the SC order parameter to develop the quantized phase winding with supercurrents flowing around the magnetic ion-hence the formation of the QAV. We have studied the binding energy for different values of $\lambda_{0}$. It turns out that the critical exchange field is only weakly dependent on $\lambda_{0}$. For example, for $\lambda_{0}=10 \mathrm{meV}, m_{0}^{c} \simeq 5.85 \mathrm{meV}$. The small decrease comes from the increased binding energy of the $j_{z}=0,|0, \pm 1 / 2\rangle$ states in Fig. 2(f), as they are located closer to the continuum due to a larger $\lambda_{0}$. In Ref. [31], it was shown that the local magnetic structure induced by the interstitial $\mathrm{Fe}$ as observed in the neutron scattering experiments, which involves about 50 lattice $\mathrm{Fe}$ atoms, can be described by a five-orbital Hubbard model with a magneticion-induced spin exchange interaction on the order of $70 \mathrm{meV}$. The intrinsic magnetic correlations in $\mathrm{Fe}(\mathrm{Te}, \mathrm{Se})$ can also renormalize the magnitude and the extent of the exchange fields induced by the magnetic impurity ion. While how the latter translate into the effective continuum theory remains to be investigated, we regard $m_{0}$ as a phenomenological parameter that controls the binding energy of the QAV.

It is instructive to estimate the line energy (tension) of the QAV, which is given by $\mathcal{E}_{\text {line }}=\left(\phi_{0} / 4 \pi \lambda_{p}\right)^{2} \ln \kappa$, where $\phi_{0}=h c / 2 e$ is the SC flux quantum, $\lambda_{p}=$ $\left(m^{*} c^{2} / 4 \pi n_{s} e^{2}\right)^{1 / 2}$ is the penetration depth, and $\kappa=$ $\lambda_{p} / \xi$ [48]. It can be written in the form

$$
\mathcal{E}_{\text {line }}=\frac{1}{3 \pi^{2}} \frac{\varepsilon_{f}^{2}}{\Delta} \frac{1}{\xi} \ln \kappa \simeq \frac{0.46}{\xi} \ln \kappa \mathrm{meV},
$$

where $\ln \kappa$ is typically of order one for type-II superconductors. For $\mathrm{Fe}(\mathrm{Te}, \mathrm{Se})$, the measured coherence length of about $2 \mathrm{~nm}$ and penetration depth of about $500 \mathrm{~nm}$ [42,49-51] provide an estimate $\mathcal{E}_{\text {line }} \sim 2.5 \mathrm{meV} / \xi$; i.e., it costs on the order of $2.5 \mathrm{meV}$ for a straight vortex line nucleated at an isolated magnetic ion to extend over a coherence length across the SC layers. Thus, for moderate QAV binding energies, a straight vortex line can only penetrate a few SC layers without encountering a "boost" by another interstitial Fe. However, the vortex lines can travel between the layers, taking advantage of the smaller line energy, and pierce through the SC planes where magnetic ions reside via the nucleation of QAVs as shown schematically in Fig. 1(b).

To develop further insights, we qualitatively elucidate the condition for the emergence of the QAV analytically. The lowering of the core energy due to the orbital exchange field is $E_{1}=\int d^{2} r \sum_{\sigma} m(r) \psi_{\sigma}^{\dagger}(r) L_{z} \psi_{\sigma}(r) / \hbar$. For an $s$-wave superconductor, the total angular momentum is quantized and given by $\hbar N / 2$ in an elemental vortex, where $N / 2$ is the number of Cooper pairs [52-55]. We thus obtain $E_{1} \simeq \pi m_{0} r_{0}^{2} n^{2 D}$, where $n^{2 D}=n d$ is the 2D particle density and $d$ the layer thickness. This is to be compared to the energy cost of the vortex line in the 2D layer $E_{2}=$ $d \mathcal{E}_{\text {line }} \simeq\left[\left(\pi \hbar^{2}\right) /\left(4 m^{*}\right)\right] n_{s}^{2 D} \ln \kappa$, where $n_{s}^{2 D}$ is the 2D superfluid density. Thus, at zero temperature, the QAV nucleates when $E_{1}>E_{2}$, i.e.,

$$
m_{0}>\frac{\hbar^{2}}{4 m^{*} r_{0}^{2}} \ln \kappa=\frac{\left|\varepsilon_{f}\right|}{2\left(r_{0} k_{f}\right)^{2}} \ln \kappa .
$$

For short-range exchange interactions $r_{0} \sim 1 / k_{f}$, the critical exchange field $m_{0}^{c}$ is therefore on the order of the Fermi energy $\left|\varepsilon_{f}\right|$, consistent with the numerical result shown above $\left(r_{0} k_{f} \simeq 1.4\right.$ for our parameters). In this sense, the small Fermi energy and superfluid density (stiffness) [56] in $\mathrm{Fe}(\mathrm{Te}, \mathrm{Se})$ favor the emergence of the QAV state. On the other hand, if the decay length $r_{0} \sim \xi$, Eq. (8) implies $m_{0}^{c} \simeq\left(\pi^{2} / 8\right)\left[\Delta^{2} /\left(\left|\varepsilon_{f}\right|\right)\right] \ln \kappa$, which is on the scale of the CdGM minigap energy and also very favorable for the nucleation of the QAV. While for superconductors in the quantum limit where $k_{f} \xi \sim 1$, such as $\mathrm{Fe}(\mathrm{Te}, \mathrm{Se})$, these two limits are essentially equivalent, it is noteworthy that even for superconductors with a substantial Fermi energy, the QAV can still be induced by a reasonable exchange interaction provided that its decay length in the low-energy effective theory is on the order of the SC coherence length, which can originate from the underlying magnetic correlations in the superconductor [57] or in the presence of sizable magnetic quantum dots.

\section{MULTIBAND AND COMPOSITE QAV}

By focusing on a single holelike band around the $\Gamma$ point in the above discussion, we have studied the physical origin and the basic properties of the QAV. We now discuss the role of the electron bands in $\mathrm{Fe}(\mathrm{Te}, \mathrm{Se})$ and demonstrate that they introduce new physics and provide a nontrivial test of the concept of the QAV. For simplicity, we ignore the weak interband Josephson coupling and consider a single isotropic electron band in the continuum limit with band mass $m_{e}^{*} \simeq 1.33 m_{e}$, Fermi energy $\varepsilon_{f}^{e} \simeq 25 \mathrm{meV}$, and pairing gap $\Delta_{e} \simeq 4 \mathrm{meV}$ extracted from the ARPES experiments [32,58-60]. The crucial observation is that for a fixed polarization of the magnetic ion with $m_{0}>0$ as before, the QAV nucleated from the electron band must preserve the chirality $\mu E_{\mu}<0$ of the vortex core states in order for the exchange field to lower its energy by pushing the CdGM states into the continuum. This requires an unchanged particle-hole vorticity $\operatorname{sgn}\left(\varepsilon_{f}^{e}\right) \nu_{e}>0$ for the anomalous vortex from the electron band. As a result, the energetically favorable QAV from the electron band must originate from the corresponding order parameter $\boldsymbol{\Delta}_{e}(\mathbf{r})=$ $\Delta_{e}(r) e^{i \nu_{e} \theta}$ with an opposite phase winding, i.e., an opposite vorticity $\nu_{e}=1$ compared to the hole band. 
In the Supplemental Material [41], we provide the details of the calculation of the QAV binding energy as a function of $m_{0}>0$ for the electron band. Since the impurity-induced SOC $\lambda_{\text {so }}$ is inversely proportional to the band mass both in sign and in magnitude $[33,34]$, we thus use $\lambda_{0}^{e}=-21 \mathrm{meV}$ for the electron band correspondingly, while keeping $r_{0}$ the same. The results show that the binding energy has an approximately linear dependence on $m_{0}$ similar to the holeband case shown in the inset of Fig. 2(g), leading to a critical $m_{0}^{c, e} \simeq 23.2 \mathrm{meV}$ beyond which the QAV emerges, consistent with the analytical expression discussed above. Thus, for a reasonably strong exchange field, composite QAVs can nucleate at the excess $\mathrm{Fe}$ sites where the supercurrents are carried by electrons from both the hole and electron bands in $\mathrm{Fe}(\mathrm{Te}, \mathrm{Se})$. Such composite QAVs can be remarkable realizations of the composite vortices with fractional flux proposed and studied both theoretically and experimentally in multiband superconductors [61-63].

\section{MAJORANA ZERO-ENERGY BOUND STATE IN QAV}

We turn to the emergence of localized MZM in the QAV when superconductivity is induced in the helical Dirac fermion TSS. In the vicinity of the magnetic ion, the effective Hamiltonian in the continuum limit, with primes indicating the TSS, can be written in the spinor basis as

$$
H^{\prime}=v_{D}(\boldsymbol{\sigma} \times \mathbf{p}) \cdot \mathbf{z}-\varepsilon_{f}^{\prime}+H_{\mathrm{soc}}^{\prime}+H_{\mathrm{ex}}^{\prime},
$$

where the velocity $v_{D}=0.216 \mathrm{eV} \cdot \AA$ and the Fermi level $\varepsilon_{f}^{\prime}=4.5 \mathrm{meV}$ above the Dirac point of the electron-doped TSS band were extracted from the ARPES experiment on $\mathrm{Fe}(\mathrm{Te}, \mathrm{Se})$ [28], as shown in Fig. 2(a). The impurity-induced $\mathrm{SOC}$ is $H_{\mathrm{soc}}^{\prime}=\lambda_{\mathrm{so}}^{\prime}(r) L_{z} \sigma_{z}$, and $H_{\mathrm{ex}}^{\prime}=-\mathcal{J}_{\mathrm{ex}}^{\prime}(r) I_{\mathrm{imp}}^{z} J_{z}$ is the exchange coupling between the TSS and the local moment [64]. In general, $\lambda_{\mathrm{so}}^{\prime}(r)$ and $\mathcal{J}_{\text {ex }}^{\prime}(r)$ can be different from those in the bulk states. We consider here the QAV formed in the hole band alone. In the corresponding BdG equation (5), the induced pairing potential for the TSS is $\boldsymbol{\Delta}^{\prime}(\mathbf{r})=\Delta_{\mathrm{QAV}}(r) e^{i \theta}$, where $\Delta_{\mathrm{QAV}}(r)$ is the pairing profile of the QAV shown in Fig. 2(e). The obtained vortexcore-states energy spectrum for the TSS is plotted in Fig. 3(a). The "isolated" bound state at $E=0$ is precisely the MZM, i.e., the $\mu^{\prime}=0$ element of the chiral CdGM states $E_{\mu^{\prime}} \propto$ $\mu^{\prime} \Delta^{2} / \varepsilon_{f}^{\prime}$, where $\mu^{\prime}=0, \pm 1, \pm 2, \ldots$ is now an integer due to the additional Berry phase of the Dirac fermions [17,41]. Note that all other CdGM states with nonzero $\mu^{\prime}$ in Fig. 3(b) obtained without coupling to the magnetic ion are pushed into the continuum above the SC gap by the exchange field via the same mechanism that produced the QAV. The gapping of the nonzero-energy CdGM states prevents the level-crossinginduced topological vortex transition [17] and protects the robustness of the MZM even for higher doping levels of the TSS.
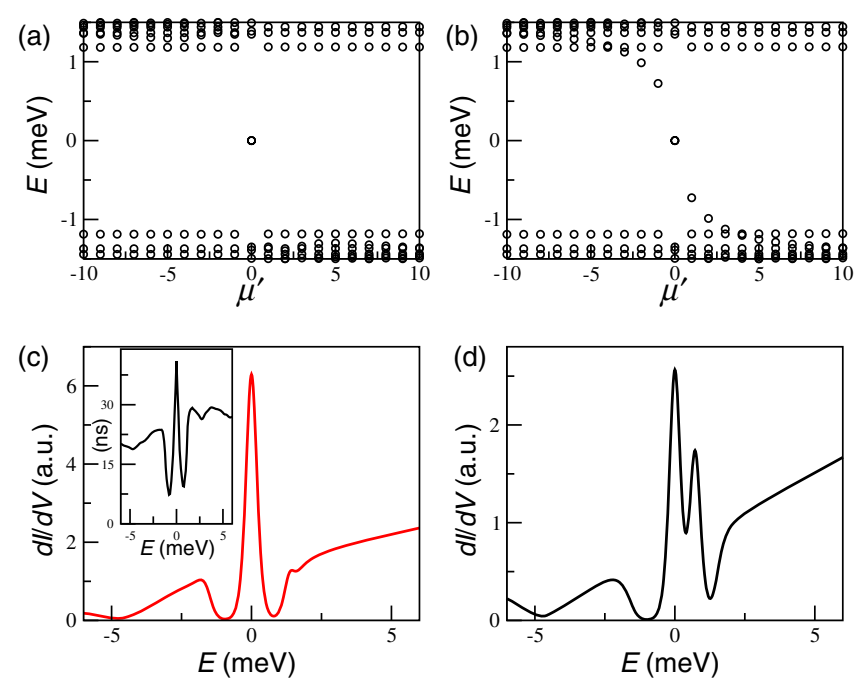

FIG. 3. The low-energy spectrum of the TSS coupled to (a) the QAV induced by the magnetic ion [see Figs. 2(e) and 2(f)], showing an isolated MZM, and (b) a conventional magneticfield-induced vortex without the magnetic ion. The parameters used for the TSS are $\lambda_{0}^{\prime}=m_{0}^{\prime}=6.6 \mathrm{meV}$. (c) The calculated tunneling conductance (local density of states) at the QAV center. Inset: STM tunneling conductance at the excess Fe site in $\mathrm{Fe}(\mathrm{Te}$, Se) [25]. (d) The tunneling conductance obtained at the center of the magnetic-field-induced vortex in (b), showing multiple CdGM vortex core states. Calculated conductance spectra are broadened by a temperature $T=1.5 \mathrm{~K}$.

There are remarkable agreements between the calculated local density of states at the center of the QAV plotted in Fig. 3(c) and the tunneling conductance measured by STM [25] at the interstitial excess $\mathrm{Fe}$ sites reproduced in the inset. Both show the $\mathrm{V}$ dip around $-4.5 \mathrm{meV}$, corresponding to the Dirac point of the TSS, the absence of coherence peaks at the gap energies $\pm \Delta$, and a spectrum free of midgap states other than the zero-bias peak. Note that without coupling to the magnetic ion, the midgap vortex core states produce multiple conductance peaks and considerably reduce the spectral weight of the MZM as shown in Fig. 3(d). Indeed, the small satellite peak at about $1.44 \mathrm{meV}$ in Fig. 3(c) comes from the CdGM states expelled into the continuum by the exchange field. It is tempting to identify a similar satellite in the STM spectrum at about $1.6 \mathrm{meV}$ with such a resonance. These findings further support the idea that the ZBS observed by STM are MZM in the QAV induced by the excess Fe. Since the QAV has trapped a SC flux quantum, flux quantization, as well as the nature of the MZM, protects the QAV and the zeroenergy bound state from external magnetic fields applied along the $c$ axis, consistent with the insensitivity of the ZBS to fields up to 8 Tesla observed by STM [25].

\section{QUANTUM ANOMALOUS VORTEX MATTER}

Let us consider a low density of dilute magnetic ions such that the SC transition temperature $T_{c}$ is reduced but 
the SC ground state remains stable. For $\mathrm{Fe}_{1+y} \mathrm{Te}_{0.55} \mathrm{Se}_{0.45}$, this is the case for excess Fe density $y<0.03$ [65]. The theory thus predicts the QAV matter illustrated in Fig. 1(b) with localized MZM at the ends of the flux lines as they enter or leave the sample's surface via the magnetic ion. Note that while the vorticity of the QAV is confined to the magnetic ion, the magnetic flux lines must be continuous. Figure 1(b) illustrates the case where the magnetic flux lines pierce through the SC layers where magnetic ions reside by the nucleation of a QAV. An immediate consequence is the appearance of QAV with opposite vorticities, i.e., both vortices and antivortices accompanied by the flipping of the local magnetic moment direction along the $c$ axis. This prediction could serve as an experimental test of the theory by spin-polarized STM and/or scanning probe SQUID.

The QAV matter is likely to have been realized in $\mathrm{Fe}(\mathrm{Te}, \mathrm{Se})$ superconductors. A crucial observation of Ref. [25] is that when two interstitial $\mathrm{Fe}$ atoms sit close together, a striking simultaneous reduction in the amplitudes of the zero-bias peaks occurs without observable energy shifts. As shown in Fig. 1(b), the continuous magnetic flux line now favors the formation of an entangled quantum anomalous vortex-antivortex pair, providing a natural explanation via the annihilation of a pair of MZM. It remains to be understood, however, why such annihilation would not cause detectable splitting of the MZM from zero energy. With increasing excess Fe concentration, the density of the QAV increases, which may provide a novel mechanism for the suppression of bulk superconductivity and the eventual superconductor-tometal quantum phase transition.

Combining the QAV matter with either SC topological surface states such as in $\mathrm{Fe}(\mathrm{Te}, \mathrm{Se})$ or a topological superconductor provides advantageous platforms in zero external magnetic fields for studying the statistics and interactions of the MZMs. In order to accomplish quantum braiding in such systems, two conditions, which are, in principle, achievable but undoubtedly challenging, need to be satisfied. First, the STM tip should only "grab and drag" the magnetic impurity ions but not the atoms in the underlying lattice to avoid drastic changes to the local electronic states. Second, the entire braiding process should be done as slowly as possible to satisfy the adiabatic condition. As long as the manipulations are in the adiabatic limit, the lowenergy Majorana bound-state description will be valid, and the non-Abelian statistics of the MZM can be realized. Furthermore, if the STM is used for both manipulating the excess Fe atoms (and the associated QAVs) and measuring the information in the MZMs, the tunneling current will introduce a source of dissipation and dephasing that puts an upper bound for the adiabatic braiding time, also accounting for the extrinsic dissipation caused by the environment. This is also true when using techniques other than the STM, as well as using other platforms such as Majorana nanowires [66]. Thus, in order to braid two MZMs successfully before the loss of coherence, the current associated with the STM needs to be sufficiently weak. An alternative approach is to separate the manipulation from the measurement processes. The MZMs can be positioned in designed arrays by the STM. Then, using the braiding in measurement-only algorithm [67], the adiabatic and coherence time constraints can be satisfied more favorably by anyonic interferometry measurements on the designed arrays.

\section{ACKNOWLEDGMENTS}

We thank Shuheng Pan, Jiaxin Yin, Andrew Potter, Hong Ding, Jiangping Hu, and Tao Xiang for valuable discussions. This work is supported by the U.S. Department of Energy, Basic Energy Sciences Grant No. DE-FG0299ER45747 (K. J. and Z. W.). K. J. also acknowledges the hospitality of Institute of Physics, Chinese Academy of Sciences under the support of the Ministry of Science and Technology of China 973 program (No. 2017 YFA0303100 and No. 2015CB921300). X. D. acknowledges the financial support from the Hong Kong Research Grants Council (Project No. GRF16300918).

[1] N. Read and D. Green, Paired States of Fermions in Two Dimensions with Breaking of Parity and Time-Reversal Symmetries and the Fractional Quantum Hall Effect, Phys. Rev. B 61, 10267 (2000).

[2] A. Kitaev, Unpaired Majorana Fermions in Quantum Wires, Phys. Usp. 44131 (2001).

[3] D. A. Ivanov, Non-Abelian Statistics of Half-Quantum Vortices in $p$-Wave Superconductors, Phys. Rev. Lett. 86, 268 (2001).

[4] C. Nayak, S. H. Simon, A. Stern, M. Freedman, and S. Das Sarma, Non-Abelian Anyons and Topological Quantum Computation, Rev. Mod. Phys. 80, 1083 (2008).

[5] L. Fu and C. L. Kane, Superconducting Proximity Effect and Majorana Fermions at the Surface of a Topological Insulator, Phys. Rev. Lett. 100, 096407 (2008).

[6] J. D. Sau, R. M. Lutchyn, S. Tewari, and S. Das Sarma, Generic New Platform for Topological Quantum Computation Using Semiconductor Heterostructures, Phys. Rev. Lett. 104, 040502 (2010).

[7] R. M. Lutchyn, J. D. Sau, and S. Das Sarma, Majorana Fermions and a Topological Phase Transition in Semiconductor-Superconductor Heterostructures, Phys. Rev. Lett. 105, 077001 (2010).

[8] Y. Oreg, G. Refael, and F. von Oppen, Helical Liquids and Majorana Bound States in Quantum Wires, Phys. Rev. Lett. 105, 177002 (2010).

[9] A. C. Potter and P. A. Lee, Multichannel Generalization of Kitaevs Majorana End States and a Practical Route to Realize Them in Thin Films, Phys. Rev. Lett. 105, 227003 (2010).

[10] X. L. Qi and S. C. Zhang, Topological Insulators and Superconductors, Rev. Mod. Phys. 83, 1057 (2011). 
[11] J. Alicea, Y. Oreg, G. Refael, F. von Oppen, and M. P. A. Fisher, Non-Abelian Statistics and Topological Quantum Information Processing in 1D Wire Networks, Nat. Phys. 7, 412 (2011).

[12] V. Mourik, K. Zuo, S. M. Frolov, S. R. Plissard, E. Bakkers, and L. P. Kouwenhoven, Signatures of Majorana Fermions in Hybrid Superconductor-Semiconductor Nanowire Devices, Science 336, 1003 (2012).

[13] S. Nadj-Perge, I. K. Drozdov, J. Li, H. Chen, S. Jeon, J. Seo, A. H. MacDonald, B. A. Bernevig, and A. Yazdani, Observation of Majorana Fermions in Ferromagnetic Atomic Chains on a Superconductor, Science 346, 602 (2014).

[14] J. P. Xu et al., Experimental Detection of a Majorana Mode in the Core of a Magnetic Vortex inside a Topological Insulator-Superconductor $\mathrm{Bi}_{2} \mathrm{Te}_{3} / \mathrm{NbSe}_{2}$ Heterostructure, Phys. Rev. Lett. 114, 017001 (2015).

[15] H. Zheng et al., Quantized Majorana Conductance, Nature (London) 556, 74 (2018).

[16] G. Xu, B. Lian, P. Tang, X.-L. Qi, and S.-C. Zhang, Topological Superconductivity on the Surface of Fe-Based Superconductors, Phys. Rev. Lett. 117, 047001 (2016).

[17] P. Hosur, P. Ghaemi, R. S. K. Mong, and A. Vishwanath, Majorana Modes at the Ends of Superconductor Vortices in Doped Topological Insulators, Phys. Rev. Lett. 107, 097001 (2011).

[18] L. Yu, Bound State in Superconductors with Paramagnetic Impurities, Acta Phys. Sin. 21, 75 (1965).

[19] H. Shiba, Classical Spins in Superconductors, Prog. Theor. Phys. 40, 435 (1968).

[20] A. I. Rusinov, Superconductivity near a Paramagnetic Impurity, JETP Lett. 9, 85 (1969).

[21] R. Yu, W. Zhang, H. J. Zhang, S. C. Zhang, X. Dai, and Z. Fang, Quantized Anomalous Hall Effect in Magnetic Topological Insulators, Science 329, 61 (2010).

[22] C. Z. Chang et al., Experimental Observation of the Quantum Anomalous Hall Effect in a Magnetic Topological Insulator, Science 340, 167 (2013).

[23] C. Caroli, P. G. de Gennes, and J. Matricon, Bound Fermion States on a Vortex Line in a Type II Superconductor, Phys. Lett. 9, 307 (1964).

[24] J. Bardeen, R. Kümmel, A. E. Jacobs, and L. Tewordt, Structure of Vortex Lines in Pure Superconductors, Phys. Rev. 187, 556 (1969).

[25] J. X. Yin et al., Observation of a Robust Zero-Energy Bound State in Iron-Based Superconductor Fe(Te,Se), Nat. Phys. 11, 543 (2015).

[26] Z. J. Wang et al., Topological Nature of the $\mathrm{FeSe}_{0.5} \mathrm{Te}_{0.5}$ Superconductor, Phys. Rev. B 92, 115119 (2015).

[27] X. X. Wu, S. Qin, Y. Liang, H. Fan, and J. Hu, Topological Characters in $\mathrm{Fe}\left(\mathrm{Te}_{1-x} \mathrm{Se}_{x}\right)$ Thin Films, Phys. Rev. B 93, 115129 (2016).

[28] P. Zhang et al., Observation of Topological Superconductivity on the Surface of an Iron-Based Superconductor, Science 360, 182 (2018).

[29] M. Y. Chen, X. Chen, H. Yang, Z. Du, X. Zhu, E. Wang, and H.-H. Wen, Discrete Energy Levels of Caroli-de GennesMatricon States in Quantum Limit in $\mathrm{FeTe}_{0.55} \mathrm{Se}_{0.45}$, Nat. Commun. 9, 970 (2018).

[30] D. F. Wang et al., Evidence for Majorana Bound States in an Iron-Based Superconductor, Science 362, 333 (2018).
[31] V. Thampy et al., Friedel-like Oscillations from Interstitial Iron in Superconducting $\mathrm{Fe}_{1+y} \mathrm{Te}_{0.62} \mathrm{Se}_{0.38}$, Phys. Rev. Lett. 108, 107002 (2012).

[32] P. Zhang et al., Multiple Topological States in Iron-Based Superconductors, Nat. Phys. 15, 41 (2019).

[33] R. J. Elliot, Theory of the Effect of Spin-Orbit Coupling on Magnetic Resonance in Some Semiconductors, Phys. Rev. 96, 266 (1954).

[34] Y. Yafet, Effective Spin-Orbit Coupling of Impurity States in Metals, J. Phys. Chem. Solids 30, 1957 (1969).

[35] J. Smit, The Spontaneous Hall Effect in Ferromagnetics I, Physica (Amsterdam) 21, 877 (1955); The Spontaneous Hall Effect in Ferromagnetics II24, 39 (1958).

[36] J. Kondo, Anomalous Hall Effect and Magnetoresistance of Ferromagnetic Metals, Prog. Theor. Phys. 27, 772 (1962).

[37] R. J. Elliott and M. F. Thorpe, Orbital Effects on Exchange Interactions, J. Appl. Phys. 39, 802 (1968).

[38] B. Coqblin and J. R. Schrieffer, Exchange Interaction in Alloys with Cerium Impurities, Phys. Rev. 185, 847 (1969).

[39] A. Fert, Skew Scattering in Alloys with Cerium Impurities, J. Phys. F 3, 2126 (1973); A. Fert and A. Friederich, Skew Scattering by Rare-Earth Impurities in Silver, Gold, and Aluminum, Phys. Rev. B 13, 397 (1976).

[40] See, e.g., J. K. Furdyna, Diluted Magnetic Semiconductors, J. Appl. Phys. 64, R29 (1988).

[41] See Supplemental Material at http://link.aps.org/ supplemental/10.1103/PhysRevX.9.011033 for more detailed discussions.

[42] H. Lei, R. Hu, E. S. Choi, J. B. Warren, and C. Petrovic, Pauli-Limited Upper Critical Field of $\mathrm{Fe}_{1+y} \mathrm{Te}_{1 x} \mathrm{Se}_{x}$, Phys. Rev. B 81, 094518 (2010).

[43] F. Gygi and M. Schluter, Self-Consistent Electronic Structure of a Vortex Line in a Type-II Superconductor, Phys. Rev. B 43, 7609 (1991).

[44] N. Hayashi, T. Isoshima, M. Ichioka, and K. Machida, LowLying Quasiparticle Excitations around a Vortex Core in Quantum Limit, Phys. Rev. Lett. 80, 2921 (1998).

[45] S. Borisenko et al., Direct Observation of Spin-Orbit Coupling in Iron-Based Superconductors, Nat. Phys. 12, 311 (2016).

[46] P. D. Johnson, H.-B. Yang, J. D. Rameau, G. D. Gu, Z.-H. Pan, T. Valla, M. Weinert, and A. V. Fedorov, Spin-Orbit Interactions and the Nematicity Observed in the Fe-Based Superconductors, Phys. Rev. Lett. 114, 167001 (2015).

[47] H. S. Greenside, E. I. Blount, and C. M. Varma, Possible Coexisting Superconducting and Magnetic States, Phys. Rev. Lett. 46, 49 (1981).

[48] P. G. de Gennes, Superconductivity of Metals and Alloys (Perseus, Cambridge, 1999).

[49] H. Kim et al., London Penetration Depth and Superfluid Density of Single-Crystalline $\mathrm{Fe}_{1+y}\left(\mathrm{Te}_{1 x} \mathrm{Se}_{x}\right)$ and $\mathrm{Fe}_{1+y}\left(\mathrm{Te}_{1 x} \mathrm{~S}_{x}\right)$, Phys. Rev. B 81, 180503(R) (2010).

[50] P. K. Biswas, G. Balakrishnan, D. M. Paul, C. V. Tomy, M. R. Lees, and A. D. Hillier, Muon-Spin-Spectroscopy Study of the Penetration Depth of $\mathrm{FeTe}_{0.5} \mathrm{Se}_{0.5}$, Phys. Rev. B 81, 092510 (2010).

[51] C. C. Homes et al., Optical Properties of the IronChalcogenide Superconductor $\mathrm{FeTe}_{0.55} \mathrm{Se}_{0.45}$, J. Phys. Chem. Solids 72, 505 (2011). 
[52] G. E. Volovik, Orbital Angular Momentum of Vortices and Textures due to Spectral Flow Through the Gap Nodes: Example of 3He-A Continuous Vortex, Pis'ma Zh. Eksp. Teor. Fiz. 61, 935 (1995).

[53] N. Nygaard, G. M. Bruun, C. W. Clark, and D. L. Feder, Microscopic Structure of a Vortex Line in a Dilute Superfluid Fermi Gas, Phys. Rev. Lett. 90, 210402 (2003).

[54] Y. Tada, W. Nie, and M. Oshikawa, Orbital Angular Momentum and Spectral Flow in Two-Dimensional Chiral Superfluids, Phys. Rev. Lett. 114, 195301 (2015).

[55] A. Prem, S. Moroz, V. Gurarie, and L. Radzihovsky, Multiply Quantized Vortices in Fermionic Superfluids: Angular Momentum, Unpaired Fermions, and Spectral Asymmetry, Phys. Rev. Lett. 119, 067003 (2017).

[56] C. C. Homes, Y. M. Dai, J. S. Wen, Z. J. Xu, and G. D. Gu, $\mathrm{FeTe}_{0.55} \mathrm{Se}_{0.45}:$ A Multiband Superconductor in the Clean and Dirty Limit, Phys. Rev. B 91, 144503 (2015).

[57] I. A. Garifullin, Yu. V. Goryunov, and G. G. Khaliullin, Study of Spin-Wave Resonance in a Superconductor with Paramagnetic Impurities, Zh. Eksp. Teor. Fiz. 104, 2414 (1993).

[58] K. Jiang, J. P. Hu, H. Ding, and Z. Q. Wang, Interatomic Coulomb Interaction and Electron Nematic Bond Order in FeSe, Phys. Rev. B 93, 115138 (2016).

[59] P. Zhang et al., Observation of Two Distinct $d_{x z} / d_{y z}$ Band Splittings in FeSe, Phys. Rev. B 91, 214503 (2015).
[60] H. Miao et al., Isotropic Superconducting Gaps with Enhanced Pairing on Electron Fermi Surfaces in $\mathrm{FeTe}_{0.55} \mathrm{Se}_{0.45}$, Phys. Rev. B 85, 094506 (2012).

[61] E. Babaev, Vortices with Fractional Flux in Two-Gap Superconductors and in Extended Faddeev Model, Phys. Rev. Lett. 89, 067001 (2002).

[62] H. Bluhm, N. C. Koshnick, M. E. Huber, and K. A. Moler, Magnetic Response of Mesoscopic Superconducting Rings with Two Order Parameters, Phys. Rev. Lett. 97, 237002 (2006).

[63] S.-Z. Lin and C. Reichhardt, Stabilizing Fractional Vortices in Multiband Superconductors with Periodic Pinning Arrays, Phys. Rev. B 87, 100508(R) (2013).

[64] Q. Liu, C.X. Liu, C. Xu, X. L. Qi, and S. C. Zhang, Magnetic Impurities on the Surface of a Topological Insulator, Phys. Rev. Lett. 102, 156603 (2009).

[65] T. J. Liu et al., Charge-Carrier Localization Induced by Excess Fe in the Superconductor $\mathrm{Fe}_{1+y} \mathrm{Te}_{1 x} \mathrm{Se}_{x}$, Phys. Rev. B 80, 174509 (2009).

[66] M. Hell, K. Flensberg, and M. Leijnse, Coupling and Braiding Majorana Bound States in Networks Defined in Two-Dimensional Electron Gases with Proximity-Induced Superconductivity, Phys. Rev. B 96, 035444 (2017).

[67] P. Bonderson, M. Freedman, and C. Nayak, MeasurementOnly Topological Quantum Computation via Anyonic Interferometry, Ann. Phys. (Amsterdam) 324, 787 (2009). 